\title{
Solidarity Behaviour for Optimizing the Waste Selective Collection
}

\author{
Eva Barrena ${ }^{1}$, David Canca ${ }^{2}$, Francisco A. Ortega ${ }^{3 *}$, Ramón Piedra-De-La-Cuadra ${ }^{2,4}$ \\ ${ }^{1}$ Department of Economics, Quantitative Methods and Economic History, Universidad Pablo de Olavide, Sevilla 41013, Spain \\ ${ }^{2}$ Department of Industrial Engineering and Management Science I, Higher Technical School of Engineering, Universidad de \\ Sevilla, Sevilla 41092, Spain \\ ${ }^{3}$ Department of Applied Mathematics I, Higher Technical School of Architecture, Universidad de Sevilla, Sevilla 41012, Spain \\ ${ }^{4}$ Department of Applied Mathematics II, Higher Technical School of Engineering, University of Seville, Seville 41092, Spain
}

Corresponding Author Email: riejos@us.es

https://doi.org/10.18280/ijsdp.150202

Received: 8 February 2019

Accepted: 10 February 2020

\section{Keywords:}

collection routes, location of waste containers, optimization, separate collection of wastes

\begin{abstract}
The problem of managing selective collection of waste using containers inside historic city centres can be performed in three sequential phases: first, locating containers along the streets; then, determining the minimum fleet size required to perform all collecting services; and finally, identifying the optimal collection routes. Obviously, the result of the first phase highly influences the procedure since this will determine the decisions to be taken for the subsequent phases. This paper is focused on the first phase. Facility-customer distances, the size of container groups and the cost of installing those containers in specific sites along the streets are parameters to be considered when designing the collecting system. Additionally, we assume that customers are willing to have a solidarity behaviour, which consists of using a container assigned to them within a pre-established proximity radius, although the assigned container may not be necessarily the closest to their residence. For this scenario, a more efficient deployment of containers for selective collection of urban solid waste can be obtained. To illustrate the performance of the developed methodology, a computational experience has been carried out on a network with randomized data based on a zone belonging to city of Seville (Spain).
\end{abstract}

\section{INTRODUCTION}

The concept of municipal waste is defined in Eurostat (2017) as those mainly produced by households, although similar wastes from sources such as commerce, offices and public institutions are included. The amount of municipal waste generated is collected by (or on behalf of) municipal authorities and disposed through a waste management system [1]. Municipal Solid Waste (MSW) includes used paper, discarded cans and bottles, food scraps, yard trimmings, and other items. Proportionally, household waste accounts for usually up to 75 per cent of all the municipal solid wastes. MSW management includes several functional phases such as waste generation, storage, collection, transportation, processing, recycling and disposal in a suitable landfill [2]. Collecting solid waste involves storage at the generation and pick-up points, pick up by the crew, trucks driving around the neighbourhood, and truck transport to a transfer station or disposal point. These tasks are difficult, complex and costly. Therefore, the objective of an efficient service should be the minimization of solid waste collection costs, together with the provision of an adequate and regular service to all of the target area [3]. Providing an efficient collection service to a city often requires a combination of techniques and equipment, to accommodate the different challenges of the various neighbourhoods within the city [4].

Waste collection and transportation phases are closely related, since the deployment of containers along the city determines both the vehicle fleet size required for picking up the collected waste into the containers and the design of efficient routes needed for that purpose. Typically, collection costs represent $80-90 \%$ and $50-80 \%$ of municipal solid waste management budget in low income and middle income countries, respectively [5]. Therefore, waste collection and transportation problems are considered as one of the most difficult operational problems when developing an integrated waste management system [6]. Eiselt and Marianov [7] provide a compilation of 64 papers that include applications throughout the world, where the main aspects of interest of the contributions have been summarized in a Table, and classified according to country, technique, criteria, objectives and type or facility to be located.

Management of solid-waste collection services is intrinsically linked to the development of effective vehicle routing (VR) models that optimize the total traveling distance of vehicles, the environmental emission and the investment costs [8]. An optimal VR is a scheduled process that allows vehicles to load waste at gather sites and dump it at a landfill by satisfying multiple objectives [9]. Through a Route optimization for Waste management (WR), both the residential routing problem and the commercial routing problem settings can be solved. Beliën et al. [10] present a review of the available literature on solid waste management problems, with a particular focus on vehicle routing problems that are classified into different categories.

Three main types of waste collection routing problems can 
be distinguished in the literature: communal site collection, container collection and kerbside collection. In the communal case, a public place is defined by the local authority in order to be share by communities for dumping their solid waste [9] The second type of collection routing problems, where full containers are collected, concerns the typical situation for industrial customers. In this case, the collection vehicles are often equipped with a specific loading mechanism and usually they can carry only one container [11]. The last collection type routing problem is the kerbside collection. In this case, householders put out their waste bins and retrieve them after the collection has been carried out by the collection fleet. In this case, the collection vehicles pass every street to pick up the garbage at a predefined date (for selective collection) or time [12].

In communal site and container collection, the collection vehicles only visit predetermined pick-up points, whereas in kerbside collection, every house needs to be visited. A second difference between both kinds of systems is that they are designed to serve different types of clients. Container collection serves industrial clients, who generally have a greater amount of waste, sometimes containing hazardous materials. On the other hand, kerbside collection, serves numerous residential customers, who typically have a small demand. Then, communal site collection and container collection problems are usually modelled as a variant of the Travelling Salesman Problem (TSP), while kerbside collection problems are typically modelled as a variant of the Chinese Postman Problem (CPP) which must include capacity constraints [6], for a detailed analysis on arc routing and node routing problems and differences.

In real scenarios, the waste collection system is distributed in a set of zones. The purpose of the zoning phase is to determine collection districts. The districts must be defined such that the total solid waste loads within each one does not exceed the capacity of the vehicles used to perform the waste collection. The problem of districting is not widely addressed in the literature, or in many cases it is assumed to be solved a priori, neglecting the influence it could have on the subsequent routing phase. Male and Liebman [13] proposed a districting heuristic based on the construction of an auxiliary graph, in which nodes represent trips and edges represent feasible trips aggregations. Eisenstein and Iyer [14] devised flexible schedules for garbage trucks in the city of Chicago. Hanafi et al. [15] studied a weekly zoning schedule problem with the aim of determining a fixed number of sectors which must be balanced with respect to the daily total waste collection time. They proposed an optimization model which can be applied to small-size instances. For large-size scenarios these authors develop a local search heuristic that is based on the definition of a zoning matrix. The proposed methods are tested on three real-world instances and 28 randomly generated instances. Labelle et al. [16] presented several models and heuristics for partitioning a city into sectors, with respect to snow disposal operations, and for assigning the sectors to disposal sites. The problem results quite similar to the problem encountered in garbage collection operations. Sahoo et al. [17] present a discussion on how to divide the area from which waste is collected into districts, with the aim of subdividing the problem, making it more manageable. Authors proposed both, a mathematical model and a two phase insertion algorithm, in which a feasible solution is first generated and later improved; see also the works of Kim et al. [18], Solomon [19] and Taillard et al. [20], which are used to address the two phase method.

Each zone has a set of starting and ending nodes associated in order to determine the tours for vehicles responsible for carrying the garbage collected in the visited containers. A planning horizon must also be considered in order to schedule a sequence of services within the useful life of each vehicle. A succession of routes (one per day, belonging to the same or to different distribution zones and performed by the same vehicle along the planning horizon) is called a circulation. Plans for determining the vehicle circulation in transportation networks are described by, for instance, Ortega et al. [21] and Canca and Barrena [22].

Community containers are the locations in the street where the waste can be transferred to the collection agency at a short distance from the dwellings where garbage is generated. Sites where community storage facilities should be located depend on the customer behaviour. If a community is willing to co-operate in their proper use by carrying their waste to the containers, rather than dropping it in the street or on open plots nearer to their homes or businesses. In these cases, the task of collection will be transferred to the street sweeping service which is more expensive than collecting from containers. A solidarity co-operation of the costumers is assumed in this paper with the goal of reducing the number of collection points

Collection vehicles visit community containers at frequent intervals, usually once daily or every second day, to remove accumulated waste. A planning horizon must also be considered in order to schedule a sequence of services within the useful life of each vehicle. The set of collecting routes, belonging to the same or to different distribution zones in the city and performed by the same vehicle along the planning horizon, is called a vehicle circulation. Plans for efficiently determining vehicle circulations in transportation networks are described by Ortega et al. [21].

Summarizing, the problem of managing selective collection of waste containers can be performed in three sequential phases devoted to: first, the location of containers along the streets; then, the determination of the minimum fleet size required to perform all collecting services; and finally, the design of optimal routes, in terms of total and equilibrated number of kilometres travelled by the trucks. The decisions to be taken in these three phases can be advised through the use of optimization models. Obviously, the result of the first phase (location of the containers) highly influences the procedure since this will determine the decisions to be taken for the subsequent phases (route of collection vehicles and service programming).

Typically, the containers should be distributed so that the distance between any two containers is not excessive. In the cities which have historic core areas it may not be possible to locate containers at the most convenient distances, because community containers can only be located along the main streets and in places where there is enough space for the container itself and for operating the collection vehicle.

The main contribution of this paper focuses on the location of collecting facilities (waste containers), and on the influence of customer solidarity behaviour on this location. For this purpose, we consider parameters such as containercustomer distances, the size of container groups, their capacities in accordance with the closest population, and the installation cost of those containers in specific sites along the streets.

The remainder of this work is organized as follows. In 
Section 2, an optimization model for locating waste containers is presented. In Section 3, a three-phases solving algorithm taking into account the characteristics of the problem and the customer solidarity behaviour is proposed. A computational experience, based on an application to a real case and distinguishing between scenarios with and without assuming customer solidarity behaviour, is implemented in Section 4. Finally, some conclusions are summarized in Section 5.

\section{MODEL FORMULATION}

We assume the following mathematical description, associated to the characteristics of the problem, that consists of a connected graph $\mathrm{G}=(\mathrm{V}, \mathrm{A})$, composed of a node set $\mathrm{V}$ (portals) and an arcs set A (directed links, i.e. arcs, representing street sections). The arcs of set A connect the nodes belonging to set $\mathrm{V}$, so that the existence of a shortest path in terms of distance or travel time between each pair of points of $\mathrm{V}$ is guaranteed. Let us suppose that set $\mathrm{V}$ contains nodes where urban waste is generated (set I) as well as points where it is possible to locate the containers to deposit them (set J). We will additionally assume that the following inclusion sequence is maintained: $J \subset I \subset V$ Note that any node $i$ of $\mathrm{V}$ located at the entrance gate of a building could be identified as a generating point of waste; in that case, node $i$ would belong to set I. Alternatively, node i could simply be a feasible site along the street, where the waste container could temporarily be located (in that case, node i would also belong to set J).

The following notation is used in order to formulate our location model for the waste containers:

- $\quad I$ : set of demand nodes $(i \in I)$. There is a population $p_{i}$ associated to each demand point $i \in I$

- $J$ : set of possible location nodes to locate waste containers $(j \in J)$. There is an upper bound $\left(c_{a p}, j \in J\right)$ in terms of capacity associated to each candidate point $j \in J$.

- $K$ : set of main types of solid waste generated in the urban area (for instance, cardboard, plastic, organic waste, scrap metal, etc. $)(k \in K)$.

Additionally, we assume a compensation cost $\beta_{j}^{k}>0$ associated with the economic value that the municipal cleaning company would be willing to pay to maintain a container of modality $k$ in node $j$ during the planning horizon. This means that the cleaning company should have previously negotiated a payment reduction with the inhabitants nearby node $j$, due to the inconveniences generated by permanently establishing the container of type $k$ close to their dwellings. In this way, if node $j$ is located in the public domain far from any building, the compensation cost could be considered to be 0 ; and, on the other hand, if the location of the container is technically unfeasible due to the proximity of a place of residence, this cost could be associated to an infinite value.

The parameters involved in our optimization model are the following:

- Each node $i$ has a known weight $w_{i}^{k}$ (which can be identified with the amount of waste in $\mathrm{kg}$ or $\mathrm{dm}^{3}$ generated in node $i$ of waste modality $k$, i.e., organic material, glass, packaging or paper units) associated.

- The shortest distances between nodes of set $V$, along network $G$, have previously been determined and recorded in the matrix $D=\left(d_{i j}\right), d_{i j} \geq 0$.
Residents associated to node $i$ would experience a displacement cost (discomfort) $C_{i j}{ }^{k}$ when having to take their type $k$ waste to the container located at point $j$. This discomfort should be limited by means of including a restriction on the maximum allowed walking distance for the users. In practice, this restriction can be modelled by the assignation of a feasible coverage radius from point $i$. A point $i$ can be considered covered by another point $j$ if the distance between them does not exceed a radius of displacement $R^{k}$. Observe that this radius is type-dependent since some types of waste may have less collecting points if its use is not as extended as others. Customers may be willing to walk longer or shorter to deposit the waste depending on its type.

Let us assume users have solidarity behavior, that is, that each customer is willing to use any container, as long as a maximum walking distance from their residence to the assigned container is not exceeded. That container might not be the closest, but this must lie within a predefined radius. In our model, a portion of inhabitants associated with node $i$ could take their garbage to the container $j$ and another portion of the population of the same node would be willing to take out their garbage to another unfilled container $j$ that is not excessively distant. This solidary behaviour of the clients would allow an efficient deployment of the containers in the area under analysis, reducing their total number and grouping them in the points of lowest cost.

Let $q_{i j}^{k} \in\{0,1\}$ be a binary auxiliary data that takes value 1 if the demand point $I$ can be covered by site $j$ by means of a container of modality $k$ (note that $q_{i j}^{k}=1$ implies that $d_{i j} 0 \leq R^{k}$ ), and 0 , otherwise. Additionally, let $N_{j}>0$ be an integer parameter that indicates the maximum number of containers that could be installed at location $j$. We assume that all containers are provided with the same capacity $Q$.

Moreover, the following variables are required in the model:

\section{Variables.}

$y_{j}^{k} \quad$ Binary variable that takes value 1, if container location $j$ is activated to collect garbage type $k$, and 0 , otherwise.

$n_{j}^{k} \quad$ Number of containers type $k$ to be installed at location $j$.

$x_{i j}^{k} \quad$ Percentage of garbage type $k$ that the client corresponding to node $i$ will deliver at location $j$.

The nature of the variables used in the model yields varied formulations to face different objectives. In our case, the following integer programming formulation determines the minimum number of container groups to be installed in the area under consideration. Note that the lower the number of garbage deposit points, the more efficient the collection procedure will be for the vehicles.

\section{Objective and constraints.}

$$
z_{1} \equiv \operatorname{Min} \sum_{j \in J} \sum_{k \in K} \beta_{j}^{k} n_{j}^{k}
$$

Subject to:

$$
\sum_{j \in J} q_{i j}^{k} y_{j}^{k} \geq 1, \quad i \in I, \quad k \in K
$$




$$
\begin{gathered}
\sum_{j \in J} q_{i j}^{k} x_{i j}^{k}=1, \quad i \in I, \quad k \in K \\
x_{i j}^{k} \leq y_{j}^{k}, \quad i \in I, \quad j \in J, \quad k \in K \\
y_{j}^{k} \leq n_{j}^{k}, \quad j \in J, \quad k \in K \\
\sum_{i \in I} w_{i}^{k} x_{i j}^{k} \leq Q \cdot n_{j}^{k} \quad j \in J, \quad k \in K \\
\sum_{k \in K} n_{j}^{k} \leq N_{j} \quad j \in J \\
y_{j}^{k} \in\{0,1\}, \quad n_{j}^{k} \in Z^{+}, \quad x_{i j}^{k} \geq 0 .
\end{gathered}
$$

The objective function (1) minimizes the cost of containers that should be installed. Note that when the radius $R^{k}$ decrease, the number of containers that can be grouped in the same location will then increase. Constraints (2) ensure that all demand for each type of waste collection is covered by the set of locations to be determined. Constraints (3) establish that the sum of coverage percentages for every demand point from the container must be equal to 1 . Constraints (4) imply that if a location is activated, then at least one container must be installed at it. Constraints (5) guarantee that if a location is not activated, then no demand point can be covered by it. Constraints (6) imply that demand points that may be served from location $j$ cannot exceed its capacity. Constraints (7) establish an upper bound on the number of containers that can be located at each site. Constraints (8) specify the domain of the variables used in the model.

Maintaining the above described constraints, an additional criterion, consisting of minimizing user travel costs, can be incorporated into the former objective by combining it with the previously considered minimization of costs in the deployment of the containers. The expression that follows formulates this double purpose:

$$
z_{2} \equiv \operatorname{Min} \sum_{i \in I} \sum_{j \in J} \sum_{k \in K} C_{i j}^{k} y_{j}^{k}+\sum_{j \in J} \sum_{k \in K} \beta_{j}^{k} n_{j}^{k}
$$

Both models (1) - (8) and (1') - (8) are of combinatorial nature and can be considered as instances of a Partial Set Covering problem [23]. The partial set covering model is NPhard since it is a generalization of the traditional location set covering problem, which is NP-hard. Cormen et al. [24] discuss the problem in detail and prove its complexity. This fact justifies the use of algorithms that provide a good heuristic solution.

A model similar to the one previously proposed has been investigated by Barrena et al. [25], in whose work heuristics were designed in a computationally feasible way and consistent with the approach. Tests carried out on randomly generated data have shown that a simple heuristic of Overflowing Deviated to Immediate Neighbourhood (ODIN) yields the best results if the inter-location spacing between adjacent containers is not excessively large. Taking these precedents into account, we propose the three-phases heuristic ODIN for solving our optimization model in order to determine the most effective deployment of waste containers along the street network.

\section{SOLVING ALGORITHM}

We propose a solving algorithm which is divided into three parts. The first phase, ODIN1, is a slightly modified version of the algorithm ODIN presented by Barrena et al. [26]. ODIN1 does not requires an initial solution and this yields a feasible solution which tends to minimize the objective function. This is done by reallocating containers that cannot be installed at their demand points to the cheapest (in terms of compensation cost) available location within radius $R^{k}$. Having into account the customer solidarity behaviour, we also propose an extension (ODIN2 and ODIN3) of this algorithm in order to minimize the number of containers at each node and to allocate them, respectively, when this change helps reducing the objective function. Allocation is then done in order to minimize the cost as well as to reduce the number of stops in subsequent phases of waste collection.

\subsection{Heuristic ODIN1}

1. Sort the points $i \in I$ that generate urban waste according to their production, from highest to lowest levels and relabel them.

2. Assign the required number of containers of type $k$ to each node $i \in I$, that is, $\boldsymbol{n}_{i}^{k}=\left\lceil\frac{w_{i}^{k}}{Q}\right\rceil$.

3. While there exists a generator point $i$ whose collection requirement exceed the established upper limit $N_{i}$ (i.e., $\sum_{k \in K} n_{i}^{k} \geq N_{i}$ ) or which does not belong to the set of possible location nodes (that is, $i \in I \backslash J$ ), do

3.1 Identify the set of possible location nodes $\operatorname{Prox}(\mathrm{i} ; \mathrm{k})$ whose distances to node $\mathrm{i}$ are less than $R^{k}$ (excluding node $\mathrm{i}$ )

3.2 Sort the nodes $j \in \operatorname{Prox}(i ; k)$ from the lowest to the highest levels according to ascending values of $\beta_{j}^{k}$

3.3 For each $j \in \operatorname{Prox}(i ; k)$ do

While $\quad\left(\sum_{k \in K} n_{i}^{k}\right)-N_{i}>0$
$N_{j}-\left(\sum_{k \in K} n_{j}^{k}\right)>0$

and

do Decrease units from $n_{i}^{k}$ and increase them in $n_{j}^{k}$

4. If all the generating points satisfy condition $\sum_{k \in K} n_{i}^{k} \leq N_{i}$

then a solution to the problem has been obtained. Otherwise, a modification of parameters $R^{k}$ or $N_{i}$ is required.

5. End.

This ODIN1 algorithm reallocates containers when demand cannot be attended at a certain node and add a container for this unattended demand at another location. However, in some occasions, there may be non-used capacity of the existing containers, and there is therefore no need to add a new one to attend demand. This give raise to propose a 
second part for this algorithm, aiming at a more efficient use of containers. This minimizes, not only compensation cost due to locations, but also the number of containers.

\subsection{Heuristic ODIN2}

In this second phase of the solution algorithm, a more efficient use of the containers is recommended. If there is enough non-used space at containers of type $\mathrm{k}$ at location $\mathrm{j}$, then the waste of type $\mathrm{k}$ is reassigned to them in order to save number of containers.

(1) For each location $j \in J$ we first calculate the number $r_{j}^{k}$ of containers of type $\mathrm{k}$ required to attend the demand from node $\mathrm{j}$ and from all others nodes whose demand is partially assigned to $\mathrm{j}$ (that is, for all $i$ such that $x_{i j}^{k} \neq 0$ ).

$$
r_{j}^{k}=\left\lceil\frac{\sum_{i \in I} x_{i j}^{k} w_{i}^{k}}{Q}\right\rceil
$$

(2) If the number of containers needed is less than the number of containers obtained from ODIN1 (that is, if $r_{j}^{k}$ $<n_{j}^{k}$ ), then diminish $n_{j}^{k}$ and update its value to $r_{j}^{k}$.

\subsection{Heuristic ODIN3}

Once all the demand is attended with the minimum number of containers (solution obtained from ODIN1 and ODIN2), we propose to redistribute them in this third phase of the algorithm. Redistributing containers to cheaper locations may help to reduce the cost, and also to reduce the number of locations with containers. Reducing the number of locations with containers will facilitate the subsequent phases of waste collection and transportation since the number of stops is reduced. This phase makes more sense in scenarios in which there is a big proportion of generating nodes which are also possible container location nodes (that is, if set $J$ is large). In these cases, it may happen that an excessive number of locations is activated and it is important to reduce them for operational tasks.

(1) Short $j \in J$ such that $n_{j}^{k} \neq 0$ from the highest to the lowest value of compensation the cost $\beta_{j}^{k}$ and re-label them.

(2) Consider the location nodes $j \in J$ that only attend demand from its own node (that is, $\sum_{i \neq j} x_{i j}^{k}=0$ ).

(3) If there exists a location node $j^{*} \in \operatorname{Prox}(j ; k) \cap J$ such that its compensation cost is lower than the one in $j$ $\left(\beta_{j^{*}}^{k}<\beta_{j}^{k}\right)$ and that can allocate more containers (that is, if $\left.\sum_{k} n_{j^{*}}^{k}<N_{j^{*}}\right)$ then increase $n_{j^{*}}^{k}$ to $\max \left\{\sum_{k} n_{j^{*}}^{k}+\right.$ $\left.n_{j}^{k}, N_{j^{*}}\right\}$ and decrease $n_{j}^{k}$ accordingly.

(4) Go to ODIN2 and Iterate until the stopping criterion is reached (when the improvement in the objective function is less or equal than a small value $\beta$ ).

\section{COMPUTATIONAL EXPERIENCE}

Our model has been tested on a graph representing a part of the street system in the city of Seville. In particular, the computational experience has been carried out on an urban area which contains a street network with 46 dwelling points (sites identified as elements of node set $I$ ). Location of nodes along the street network and internode distances are illustrated in Figure 1.

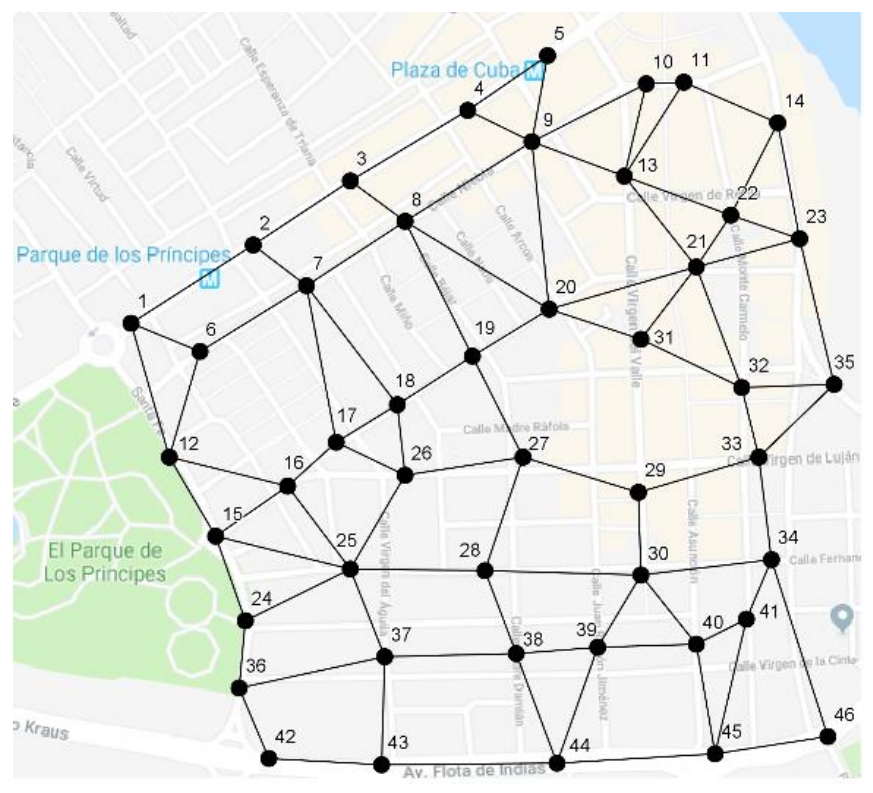

Figure 1. A network of 46 nodes depicting an area of Seville

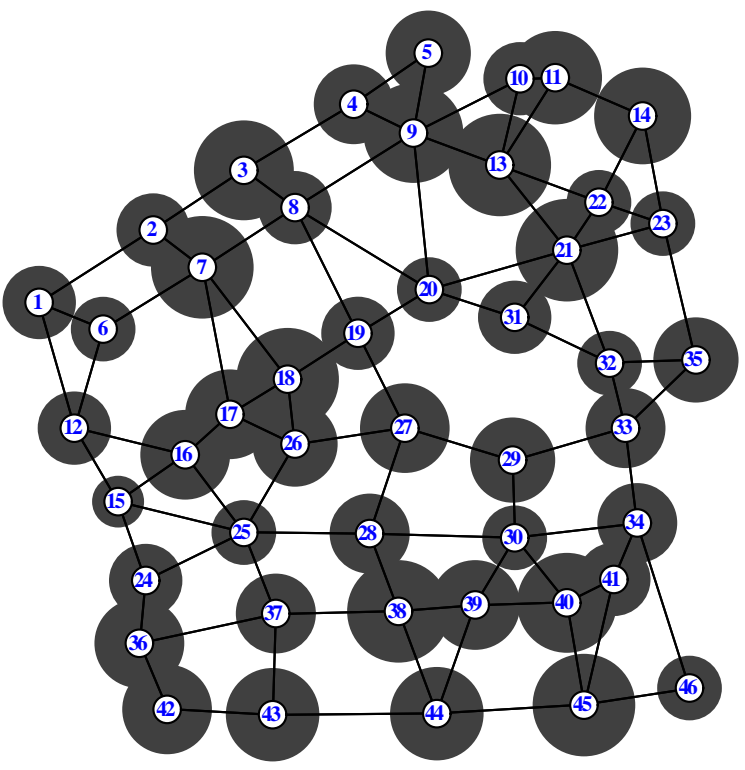

Figure 2. Container distribution with 46 nodes

The amount of daily produced waste has been randomised within the interval $[400 \mathrm{~kg}, 5000 \mathrm{~kg}]$. By considering homogeneous containers of capacity $500 \mathrm{~kg}$, it is possible to initially assign to each residential place a container cluster (located at this same site) whose amount varies between 1 and 10 . In order to adapt to a real context, a limitation on the number of containers that share the same geographical location has been set to 10 . Additionally, a monthly unit cost for locating a container at each site $j$ must be considered. In the experiment, this cost has been considered as random within the interval $[0,10]$ measured in $€$.

In the baseline scenario where no optimization procedure was applied, the 236 containers needed to collect all the produced garbage would involve a cost of $1149 €$ per month. In Figure 2, the container clusters, which are needed to 
guarantee the collection of all urban waste by means of user displacements to the deposit point nearer than $\mathrm{R}=100 \mathrm{~m}$, are represented by circles of variable radius between 1 and 10 . The radius of each circle is proportional to the size of the corresponding container group.

On the other hand, we also consider a first scenario assuming customer solidarity behaviour, that is, that customers are willing to carry their garbage to their specifically assigned containers (not necessarily to the closest container to their place of residence), within a pre-established proximity radius of $\mathrm{R}=100 \mathrm{~m}$. In this scenario, a more efficient distribution of the containers can be obtained by means of the optimization model previously proposed (ODIN 1-3). Two sub-scenarios have been analysed according to size $(N)$ of the container group at the same point.

For $N=6$, the proposed methodology yields the following results:

The number of initial container groups (46) can be reduced to 43 .

- The compensation cost of the maintenance of the 236 containers on the street, is reduced by 10.01 percent. The monthly cost associated to the solution shown in Figure 3 is now 1034 euros.

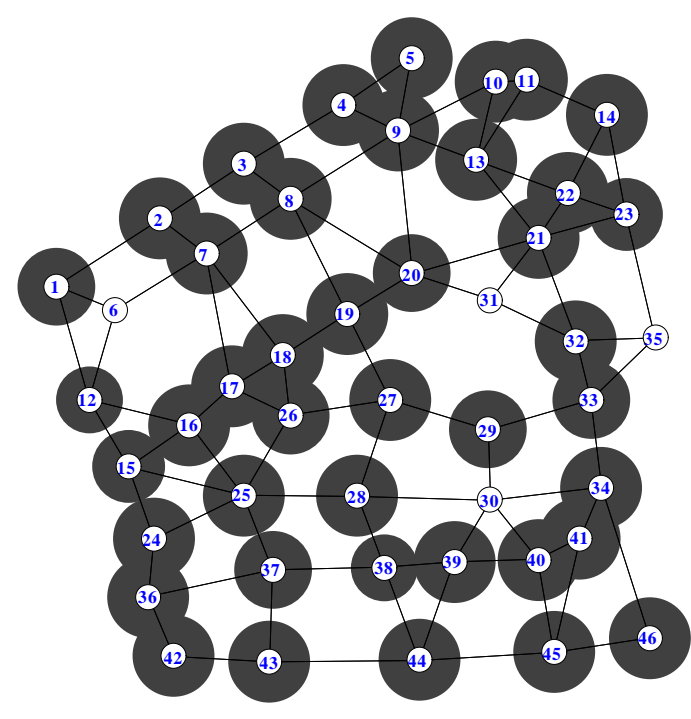

Figure 3. Container distribution with 43 nodes

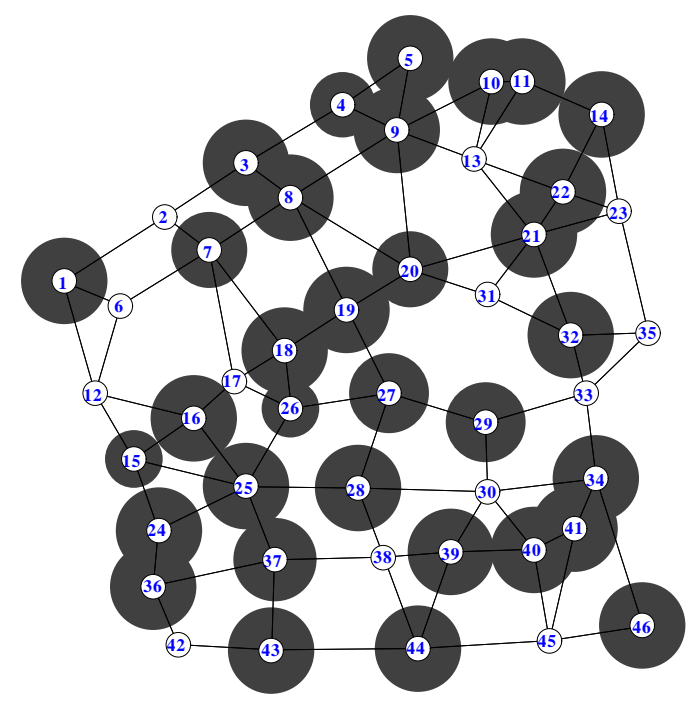

Figure 4. Container distribution with 33 nodes
For $N=8$, the proposed methodology yields the following results:

The number of initial container groups (46) can be reduced to 33 .

- The compensation cost of the maintenance of the 236 containers on the street, is reduced by 29.42 percent. The monthly cost associated to the solution shown in Figure 4 is now 811 euros.

In a second scenario, we consider a selective collection of solid waste by using three different types of containers (a situation like the one shown in the Figure 5).

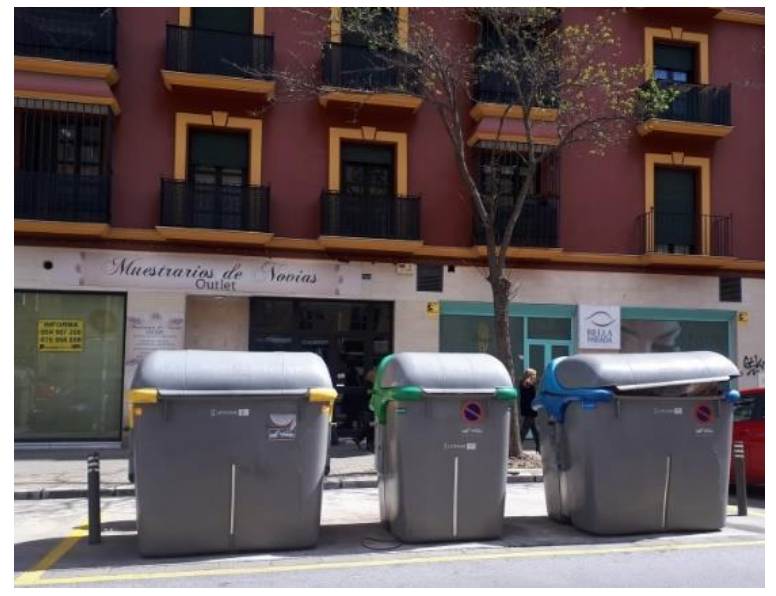

Figure 5. An example of three different types of solid waste collection

For this case, the total amount of waste generated at each node coincides with that of the first scenario, but its distribution in each of the three types of waste considered has been randomly generated. The requirement to separately store the waste yields an increment in the number of containers needed to carry out the collection of waste. This increment with respect to the first scenario, which in the experiment is equal to $22.03 \%$ (288 containers now, versus 236 in scenario 1), leads to a redistribution of the containers. A new application of the ODIN heuristics, maintaining the values established for the parameters $R^{k}$ and $N_{j}$, provides a more efficient distribution of the containers, since those ones can be grouped in 41 places, instead of the initial 46 nodes.

\section{CONCLUSIONS}

A methodology for the deployment of containers for selective collection of urban solid waste has been proposed in this work. The mathematical optimization model formulated for this purpose has been identified as a version of the Partial Set Covering problem, whose computational complexity motivates the use of heuristics to face large real-life scenarios. Following that recommendation, a three-phase greedy algorithm of overflowing deviated to the immediate neighbourhood has been developed to solve the proposed mathematical programming model. This algorithm takes into account the characteristics of the problem and it specially considers the customer solidarity behaviour.

In order to illustrate the performance of the developed methodology, a computational experience has been carried out on an urban system composed of 46 nodes with randomised data based on a zone belonging to the area of Seville (Spain). Apart from the baseline scenario, two 
different scenarios are considered by assuming that customers have solidarity behaviour, as they commit to deposit their waste in containers that are not necessarily the closest to their homes.

The first scenario maintains the current non-selective collection of urban solid waste and considers different options by varying the size of the container group at the same point. After the optimization procedure for the biggest size considered, the compensation cost is reduced by $14.48 \%$ and the number of clusters is reduced from 46 to 33 (decrease of $29.42 \%$ ), thus facilitating the subsequent phases of service programming and collection route design. The second scenario incorporates the selective collection, thus yielding an increment on the number of containers but, even though, the number of nodes is reduced to 41 (decrease of $10.86 \%$ ). The evaluation of two generated scenarios illustrates then that the methodology meets the objective of efficiently designing a deployment of containers for selective collection of urban solid waste.

We must conclude that the optimization of sites, where community storage facilities should be located highly, depends on the customer behaviour. The number of containers and therefore the cost associated with their location and transportation can be significantly reduced if a community is willing to co-operate by carrying their waste to the appropriate containers within a predefined radius, even if eventually these are not the nearest to their residence. A solidarity co-operation of the costumers is assumed in this paper with the goal of reducing the number of collection points.

\section{ACKNOWLEDGMENT}

This research has been partially supported by the Spanish Ministry of Science and Education through grants MTM2015-67706-P (MICINN, Spain) and FQM-241 (Junta de Andalucía, Spain), and finally by the FEDER funds of the European Union. This support is gratefully acknowledged.

\section{REFERENCES}

[1] European Commission. Guidance on municipal waste data collection May 2017. Eurostat - Unit E2 Environmental statistics and accounts; sustainable development.

https://ec.europa.eu/eurostat/documents/342366/351811 /Municipal+Waste+guidance, accessed on Feb. 8, 2019.

[2] Khan, D., Samadder, S.R. (2014). Municipal solid waste management using geographical information system aided methods: A mini review. Waste Management \& Research, 32(11): 1049-1062. https://doi.org/10.1177/0734242X14554644

[3] U.S. Congress, Office of Technology Assessment. (1989). Facing America's Trash: What Next for Municipal Solid Waste, OTA-O-424. Washington, DC: U.S. Government Printing Office.

[4] Coffey, M., Coad, A. (2010). Collection of Municipal Solid Waste in Developing Countries. United Nations Human Settlements Programme (UN-HABITAT). Gutenberg Press, Malta.

[5] Aremu, A.S. (2013). In-town tour optimization of conventional mode for municipal solid waste collection.
Nigerian Journal of Technology, 32(3): 443-449

[6] Nuortio, T., Kytöjoki, J., Niska, H., Bräysy, O. (2006). Improved route planning and scheduling of waste collection and transport. Expert Systems with Applications, $30(2)$ : 223-232.

https://doi.org/10.1016/j.eswa.2005.07.009

[7] Eiselt, H.A., Marianov, V. (2015). Location modeling for municipal solid waste facilities. Computers \& Operations Research, 62:

https://doi.org/10.1016/j.cor.2014.05.003

[8] Apaydin, O., Gonullu, M.T. (2011). Route time estimation of solid waste collection vehicles based on population density. Global Nest Journal, 13(2): 162-169. https://doi.org/10.30955/gnj.000739

[9] Tung, D.V., Pinnoi, A. (2000). Vehicle routingscheduling for waste collection in Hanoi. European Journal of Operational Research, 125(3): 449-468. https://doi.org/10.1016/S0377-2217(99)00408-7

[10] Beliën, J., De Boeck, L., Van Ackere, J. (2014). Municipal solid waste collection and management problems: A literature review. Transportation Science, 48(1): 78-102. https://doi.org/10.1287/trsc.1120.0448

[11] Bodin, L., Mingozzi, A., Baldacci, R., Ball, M. (2000). The rollon-rolloff vehicle routing problem. Transportation Science, 34(3): 271-288. https://doi.org/10.1287/trsc.34.3.271.12301

[12] Sniezek, J., Bodin, L. (2006). Using mixed integer programming for solving the capacitated arc routing problem with vehicle/site dependencies with an application to the routing of residential sanitation collection vehicles. Annals of Operations Research, 144 33-58. https://doi.org/10.1007/s10479-006-0006-y

[13] Male, J.W., Liebman J.C. (1978). Districting and routing for solid waste collection. Journal of Environmental Engineering Division, 104: 1-14.

[14] Eisenstein, D.D., Iyer, A.V. (1997). Garbage collection in Chicago: A dynamic scheduling model. Management Science, 43(7): 922-33. https://doi.org/10.1287/mnsc.43.7.922

[15] Hanafi, S., Freville, A., Vaca, P. (1999). Municipal solid waste collection: An effective data structure for solving the sectorization problem with local search methods. INFOR: Information Systems and Operational Research, 37: 236-54. https://doi.org/10.1080/03155986.1999.11732383

[16] Labelle, A., Langevin, A., Campbell. J.F. (2002). Sector design for snow removal and disposal in urban areas. Socio-Economic Planning Sciences, 36: 183-202. https://doi.org/10.1016/S0038-0121(01)00024-6

[17] Sahoo, S., Kim, S., Kim, B., Kraas, B., Popov, A. (2005). Routing optimization for waste management. Interfaces, $35:$ 24-36. https://doi.org/10.1287/inte.1040.0109

[18] Kim, B.I., Kim, S., Sahoo, S. (2004). Balanced clustering algorithms for vehicle routing problems. In: Proceedings of the Institute of Industrial Engineers annual conference. Houston, USA.

[19] Solomon, M.M. (1997). Algorithms for the vehicle routing and scheduling problems with time windows. Operations Research, 35: 254-65. https://doi.org/10.1287/opre.35.2.254

[20] Taillard, E., Badeau, P., Gendreau, M., Guertin, F., Potvin, J.Y. (1997). A tabu search heuristic for the vehicle routing problem with soft time windows. 
Transportation $\quad$ Science, 31: 70-86. https://doi.org/10.1287/trsc.31.2.170

[21] Ortega, F.A., Barrena, E., Canca, D. (2016). Improving circulation plans of rolling stock in railway networks by means of the optimal location of depots. XXIII EURO Working Group on Locational Analysis, Malaga, Spain.

[22] Canca, D., Barrena, E. (2018). The integrated rolling stock circulation and depot location problem in railway rapid transit systems. Transportation Research. Part E: Logistics and Transportation Review, 109: 115-138. https://doi.org/10.1016/j.tre.2017.10.018

[23] Daskin, M.S., Owen, S.H. (1999). Two new location covering problems: The partial P-center problem and the partial set covering problem. Geographical Analysis, 31(3):

217-235.

https://doi.org/10.1111/gean.1999.31.1.217
[24] Cormen, T.H., Leiserson, C.E., Rivest, R.L. (1991). Introduction to Algorithms. The MIT Press.

[25] Barrena, E., Canca, D., Ortega, F.A. (2017). Routing vehicle fleets during disaster relief. Proceedings of the VIII International Workshop on Locational Analysis and Related Problems (2017). Edited by Marta Baldomero-Naranjo, Inmaculada Espejo-Miranda, Luisa I. Martínez-Merino, Antonio M. Rodríguez-Chía, Diego Ruiz-Hernández. ISBN 978-84-697-5263-0, pp. 15-16. Segovia (Spain).

[26] Barrena, E., Canca, D., Ortega, F.A. (2018). Piedra-dela-Cuadra, R., Optimizing Container Location for Selective Collection of Urban Solid Waste. Chapter at book Waste Management and the Environment IX, Edited by F.A. Ortega, M. Lega and H. Itoh. WIT Press, Southampton, UK, pp. 1-10. 